\title{
Invariant Manifolds and Grobman-Hartman Theorem for Equations with Degenerate Operator at the Derivative*
}

\author{
Bülent Karasözen ${ }^{1}$, Irina Konopleva, and Boris Loginov ${ }^{2}$ \\ 1 Ankara Middle-East Technical University, 06531 Ankara, Turkey, \\ bulent@metu.edu.tr \\ 2 Ulyanovsk State Technical University, 432027 Ulyanovsk, Russia, \\ i.konopleva@ulstu.ru, loginov@ulstu.ru
}

\begin{abstract}
Analog of Grobman-Hartman theorem about stable and unstable manifolds solutions for differential equations in Banach spaces with degenerate Fredholm operator at the derivative are proved. In contrast to usual evolution equation here central manifold arises even in the case of spectrum absence on the imaginary axis. Jordan chains tools and implicit operator theorem are used. The obtained results allow to develop center manifold methods for computation of bifurcation solution asymptotics and their stability investigation.
\end{abstract}

\section{Introduction}

Branching theory of solutions of nonlinear equations has various applications in scientific computing [1, [2], 3]. This is one of the areas in applied mathematics intensively developing in last fifty years. The goals of this theory are the qualitative theory of dynamical systems [3], computation of their solutions [4] under absence of conditions guaranteeing the uniqueness of the solutions. The classical Lyapounov-Schmidt method even in contemporary presentation [5] often insufficient for computation of complicated dynamics, like bifurcation to invariant tori. Therefore in the last two decades the center manifold theory [6] and methods are developed. However this theory is completely absent for evolution equations with degenerate operator at the derivative, having numerous applications in filtration theory [7, nonlinear waves theory (the Boussinesq-Love equation) [8] and motion theory of non-Newtonian fluids [9].

The presented work is devoted to invariant manifolds technique and presents the introduction to center manifold methods for evolution equations with Fredholm operator at the derivative. It has found some applications to investigation of the bifurcating solutions stability [10. The second section of this article contains the necessary tools of generalized Jordan chains, the third, fourth and fifth ones - some aspects of invariant manifolds theory and Grobman-Hartman

\footnotetext{
* Supported by NATO-TÜBITAK PC program and Russian Foundation for Basic Research (project No: 01-01-0019)
} 
theorem analogs for such equations. Here the nontrivial center manifold arises even at the absence of $\sigma_{A}(B)$ spectrum on the imaginary axis. It is considered also the simple case of $\sigma_{A}(B)$ presence on imaginary axis (section 4 ). For the computation of center manifold in section 3 successive approximation method is suggested.

\section{Generalized Jordan Chains Tools}

Let $E_{1}$ and $E_{2}$ be Banach spaces, $A: E_{1} \supset D_{A} \rightarrow E_{2}, B: E_{1} \supset D_{B} \rightarrow E_{2}$ be densely defined closed linear Fredholm operators, where $D_{B} \subset D_{A}$ and $A$ is subordinated to $B$ (i.e. $\|A x\| \leq\|B x\|+\|x\|$ on $D_{B}$ ) or $D_{A} \subset D_{B}$ and $B$ is subordinated to $A$ (i.e. $\|B x\| \leq\|A x\|+\|x\|$ on $D_{A}$ ). The differential equation

$$
A \frac{d x}{d t}=B x-R(x), \quad R(0)=0, \quad R_{x}(0)=0
$$

is considered.

It is known [5,1012 that for the zero-subspaces of the operators $A$ and $B$ $\mathcal{N}(A)=\operatorname{span}\left\{\phi_{1}, \ldots, \phi_{m}\right\}, \mathcal{N}(B)=\operatorname{span}\left\{\varphi_{1}, \ldots, \varphi_{n}\right\}, \mathcal{N}(A) \cap \mathcal{N}(B)=\{0\}$ and defect-subspaces $\mathcal{N}^{*}(A)=\operatorname{span}\left\{\hat{\psi}_{1}, \ldots, \hat{\psi}_{m}\right\}, \mathcal{N}^{*}(B)=\operatorname{span}\left\{\psi_{1}, \ldots, \psi_{n}\right\}$, the biorthogonal systems $\left\{\vartheta_{j}\right\}_{1}^{m},<\phi_{i}, \vartheta_{j}>=\delta_{i j} ;\left\{\zeta_{j}\right\}_{1}^{m},<\zeta_{i}, \hat{\psi}_{j}>=\delta_{i j}$ and $\left\{\gamma_{j}\right\}_{1}^{n},<\varphi_{i}, \gamma_{j}>=\delta_{i j} ;\left\{z_{j}\right\}_{1}^{n},<z_{i}, \psi_{j}>=\delta_{i j}$ can be chosen so that the following biorthogonality conditions for the corresponding Jordan chains $\left(\left\{\phi_{i}^{(s)}\right\}, s=1, \ldots, q_{i}, \phi_{i}^{(1)}=\phi_{i}, A \phi_{i}^{(s)}=B \phi_{i}^{(s-1)},<\phi_{i}^{(s)}, \vartheta_{j}>=\right.$ $0, s=2, \ldots, q_{i}, i, j=1, \ldots, m, D_{q} \equiv \operatorname{det}\left[<B \phi_{i}^{\left(q_{i}\right)}, \hat{\psi}_{j}>\right] \neq 0 ;\left\{\varphi_{i}^{(s)}\right\}, s=$ $1, \ldots, p_{i}, \varphi_{i}^{(1)}=\varphi_{i}, \varphi_{i}^{(s)}=A \varphi_{i}^{(s-1)},<\varphi_{i}^{(s)}, \gamma_{j}>=0, s=2, \cdots, p_{i}, \quad i, j=$ $1, \ldots, n, \quad D_{p} \equiv \operatorname{det}\left[\left\langle A \varphi_{i}^{\left(p_{i}\right)}, \psi_{j}\right\rangle\right] \neq 0$; for adjoint operator-functions $A^{*}-\lambda B^{*}$ and $B^{*}-\mu A^{*}$ Jordan chains $\left\{\hat{\psi}_{j}^{(s)}\right\}, s=1, \ldots, q_{i}, i=1, \ldots, m$, and $\left\{\psi_{j}^{(s)}\right\}, s=1, \ldots, p_{j}, j=1, \ldots, n$ are defined analogously ) would be satisfied:

$$
\begin{gathered}
<\phi_{i}^{(j)}, \vartheta_{k}^{(\ell)}>=\delta_{i k} \delta_{j \ell},<\zeta_{i}^{(j)}, \hat{\psi}_{k}^{(\ell)}>=\delta_{i k} \delta_{j \ell}, j(\ell)=1, \ldots, q_{i}\left(q_{k}\right), \\
\vartheta_{k}^{(\ell)}=B^{*} \hat{\psi}_{k}^{\left(q_{k}+1-\ell\right)}, \zeta_{i}^{(j)}=B \phi_{i}^{\left(q_{i}+1-j\right)}, i, k=1, \ldots, m \\
<\varphi_{i}^{(j)}, \gamma_{k}^{(\ell)}>=\delta_{i k} \delta_{j \ell},<z_{i}^{(j)}, \psi_{k}^{(\ell)}>=\delta_{i k} \delta_{j \ell}, j(\ell)=1, \ldots, p_{i}\left(p_{k}\right) \\
\gamma_{k}^{(\ell)}=A^{*} \psi_{k}^{\left(p_{k}+1-\ell\right)}, z_{i}^{(j)}=A \varphi_{i}^{\left(p_{i}+1-j\right)}, i, k=1, \ldots, n
\end{gathered}
$$

The relations (2), (3) allow to introduce 512 the projectors on the rootsubspaces $K(A ; B)=\operatorname{span}\left\{\phi_{i}^{(s)}\right\}\left(k_{A}=\sum_{i=1}^{m} q_{i}=\operatorname{dim} K(A ; B)\right.$ is the rootnumber for $A-\lambda B)$ and $K(B ; A)=\operatorname{span}\left\{\varphi_{i}^{(s)}\right\}\left(k_{B}=\sum_{i=1}^{n} p_{i}\right.$-the root-number for $B-\mu A)$ : 


$$
\begin{aligned}
& \mathbf{p}=\sum_{i=1}^{m} \sum_{j=1}^{q_{i}}<\cdot, \vartheta_{i}^{(j)}>\phi_{i}^{(j)}=<\cdot,>\phi: E_{1} \rightarrow E_{1}^{k_{A}}=K(A ; B), \\
& \mathbf{q}=\sum_{i=1}^{m} \sum_{j=1}^{q_{i}}<\cdot, \hat{\psi}_{i}^{(j)}>\zeta_{i}^{(j)}=<\cdot, \hat{\psi}>\zeta: E_{2} \rightarrow E_{2, k_{A}}=\operatorname{span}\left\{\zeta_{i}^{(j)}\right\}, \\
& \mathbf{P}=\sum_{i=1}^{n} \sum_{j=1}^{p_{i}}<\cdot, \gamma_{i}^{(j)}>\varphi_{i}^{(j)}=<\cdot, \gamma>\varphi: E_{1} \rightarrow E_{1}^{k_{B}}=K(B ; A), \\
& \mathbf{Q}=\sum_{i=1}^{n} \sum_{j=1}^{p_{i}}<\cdot, \psi_{i}^{(j)}>z_{i}^{(j)}=<\cdot, \psi>z: E_{2} \rightarrow E_{2, k_{B}}=\operatorname{span}\left\{z_{i}^{(j)}\right\}
\end{aligned}
$$

(where $\phi=\left(\phi_{1}^{(1)}, \cdots, \phi_{1}^{\left(q_{1}\right)}, \cdots, \phi_{m}^{(1)}, \cdots, \phi_{m}^{\left(q_{m}\right)}\right)$, the vectors $\vartheta, \hat{\psi}, \zeta$ and $\varphi, \gamma, \psi, z$ are defined analogously ), generating the following direct sums expansions

$$
\begin{aligned}
& E_{1}=E_{1}^{k_{A}} \dot{+} E_{1}^{\infty-k_{A}}, E_{2}=E_{2, k_{A}} \dot{+} E_{2, \infty-k_{A}} \\
& E_{1}=E_{1}^{k_{B}} \dot{+} E_{1}^{\infty-k_{B}}, E_{2}=E_{2, k_{B}} \dot{+} E_{2, \infty-k_{B}} .
\end{aligned}
$$

The $\mathbf{p}, \mathbf{q}^{-}$and $\mathbf{P}, \mathbf{Q}$-intertwining relations are realized

$$
\begin{aligned}
& A \mathbf{p}=\mathbf{q} A \text { on } D_{A}, B \mathbf{p}=\mathbf{q} B \text { on } D_{B} ; B \mathbf{P}=\mathbf{Q} B \text { on } D_{B}, A \mathbf{P}=\mathbf{Q} A \text { on } D_{A}, \\
& A \phi=\mathfrak{A}_{A} \zeta, B \phi=\mathfrak{A}_{B} \zeta, B^{*} \hat{\psi}=\mathfrak{A}_{B} \vartheta ; B \varphi=\mathcal{A}_{B} z, A \varphi=\mathcal{A}_{A} z, A^{*} \psi=\mathcal{A}_{A} \gamma
\end{aligned}
$$

with cell-diagonal matrices $\mathfrak{A}_{A}=\left(A_{1}, \ldots, A_{m}\right), \mathfrak{A}_{B}=\left(B_{1}, \ldots, B_{m}\right) ; \mathcal{A}_{B}=$ $\left(B^{1}, \ldots, B^{n}\right), \mathcal{A}_{A}=\left(A^{1}, \ldots, A^{n}\right)$, where $q_{i} \times q_{i}$-cells $\left(p_{i} \times p_{i}\right.$-cells $)$ have the forms

$$
A_{i}=\left(\begin{array}{cccccc}
0 & 0 & 0 & \ldots & 0 & 0 \\
0 & 0 & 0 & \ldots & 0 & 1 \\
\vdots & \vdots & \vdots & \ddots & \vdots & \vdots \\
0 & 0 & 1 & \ldots & 0 & 0 \\
0 & 1 & 0 & \ldots & 0 & 0
\end{array}\right), \quad B_{i}=\left(\begin{array}{cccccc}
0 & 0 & 0 & \ldots & 0 & 1 \\
0 & 0 & 0 & \ldots & 1 & 0 \\
\vdots & \vdots & \vdots & \ddots & \vdots & \vdots \\
0 & 1 & 0 & \ldots & 0 & 0 \\
1 & 0 & 0 & \ldots & 0 & 0
\end{array}\right)
$$

( $B^{i}$ is of the type $A_{i}$ and $A^{i}$ has the form of $B_{i}$ ). Here

$$
\begin{gathered}
\mathcal{N}(A) \subset E_{1}^{k_{A}}, A E_{1}^{k_{A}} \subset E_{2, k_{A}}, \quad A\left(E_{1}^{\infty-k_{A}} \cap D_{A}\right) \subset E_{2, \infty-k_{A}}, \\
\mathcal{N}(B) \subset E_{1}^{\infty-k_{A}}, B E_{1}^{k_{A}} \subset E_{2, k_{A}}, \quad B\left(E_{1}^{\infty-k_{A}} \cap D_{B}\right) \subset E_{2, \infty-k_{A}}
\end{gathered}
$$

$\stackrel{\sqcap}{A}=\left.A\right|_{E_{1}^{\infty-k_{A}} \cap D_{A}}, \stackrel{\sqcap}{B}=\left.B\right|_{E_{1}^{\infty-k_{A}} \cap D_{B}}$ and the mappings $B: E_{1}^{k_{A}} \rightarrow E_{2, k_{A}}, \stackrel{\sqcap}{A}$ : $E_{1}^{\infty-k_{A}} \cap D_{A} \rightarrow E_{2, \infty-k_{A}}$ are one-to-one. Analogously, the operators $B$ and $A$ act in invariant pairs of the subspaces $E_{1}^{k_{B}}, E_{2, k_{B}}$ and $E_{1}^{\infty-k_{B}}, E_{2, \infty-k_{B}}$ and also $\stackrel{\sqcup}{B}=\left.B\right|_{E_{1}^{\infty-k_{B}} \cap D_{B}}: E_{1}^{\infty-k_{B}} \cap D_{B} \rightarrow E_{2, \infty-k_{B}}, A: E_{1}^{k_{B}} \rightarrow E_{2, k_{B}}$ are isomorphisms.

\section{Analogs of Grobman-Hartman Theorem at $\sigma_{A}^{0}(B)=\varnothing$}

It is supposed that for the $A$-spectrum $\sigma_{A}(B)$ of the operator $B \operatorname{Re} \sigma_{A}(B) \neq 0$ and the spectral sets $\sigma_{A}^{-}(B)=\left\{\mu \in \sigma_{A}(B) \mid \operatorname{Re} \mu<0\right\}$ and $\sigma_{A}^{+}(B)=\{\mu \in$ $\left.\sigma_{A}(B) \mid \operatorname{Re} \mu>0\right\}$ be distant from the imaginary axis on some distance $d>0$. 
All solutions of the corresponding to (1) linear Cauchy problem

$$
A \frac{d x}{d t}=B x, \quad x(0)=x_{0}
$$

belong to $E_{1}^{\infty-k_{A}}$ and (8) is solvable iff $x_{0} \in E_{1}^{\infty-k_{A}}$. In fact, one sets $x=v+w$, $v(t)=\sum_{i=1}^{m} \sum_{s=1}^{q_{i}} \xi_{i s}(t) \phi_{i}^{(s)} \in E_{1}^{k_{A}}, w(t) \in E_{1}^{\infty-k_{A}}$, then (8) is splitting into the system

$$
\frac{d \xi_{i s}(t)}{d t}=\xi_{i, s-1}, s=2, \ldots, q_{i}, i=1, \ldots, m, \quad \xi_{i q_{i}}=0 ; \stackrel{\sqcap}{A} \frac{d w}{d t}=\stackrel{\sqcap}{B} w .
$$

Consequently $\xi_{\text {is }}(t)=0$, solution of (8) takes the form

$$
x(t)=\exp \left(\stackrel{\sqcap^{-1}}{\stackrel{\cap}{B}} t\right) x_{0}, \quad x_{0} \in E_{1}^{\infty-k_{A}}
$$

and $\sigma_{A}(B)=\sigma\left(\stackrel{ }{A}^{-1} \stackrel{\mapsto}{B}\right)$. Here the function $\exp \left(\stackrel{\sqcap}{A}^{-1} \stackrel{\sqcap}{B} t\right)$ has the form of the contour integral $\frac{1}{2 \pi i} \int_{\gamma}\left(\mu I-\stackrel{\cap^{-1}}{A} \stackrel{\sqcap}{B}\right)^{-1} e^{\mu t} d t$ at the assumption about sectorial property [3] of the operator $\stackrel{\sqcap^{-1}}{A} \stackrel{\sqcap}{B}$ (or, that is the same, about $A$-sectorial property of the operator $B$ [13]) with some special contour $\gamma$ belonging to sector $S_{\alpha, \theta}(B)$ in $A$-resolvent set of the operator $B[13]$.

The more so, this is true when the operator $\AA^{-1} \stackrel{\sqcap}{B}$ is bounded.

At the generalization of the Grobman-Hartman theorem we will follow to the work [1]. Let us define the spaces $D_{k}, k=1,2$ with graphs norms:

$1^{0} . D_{1}=D_{B} \subset D_{A}$ with the norm $\|x\|_{1}=\|x\|_{E_{1}}+\|B x\|_{E_{2}}, x \in D_{1}$, if $A$ is subordinated to $B$,

$2^{0} . D_{2}=D_{A} \subset D_{B}$ with the norm $\|x\|_{2}=\|x\|_{E_{1}}+\|A x\|_{E_{2}}, x \in D_{2}$, if $B$ is subordinated to $A$,

and introduce the spaces $X_{k 0}, X_{k 1}, X_{k 2}, Y_{k 0}, Y_{k 1}, Y_{k 2}$ consisting of the bounded uniformly continuous functions $f(t)$ on $[0, \infty)$ with their values correspondingly in $D_{k}, D_{k} \cap E_{1}^{\infty-k_{A}}, E_{1}^{k_{A}}, E_{2}, E_{2, \infty-k_{A}}, E_{2, k_{A}}$ with supremum norms on the relevant spaces, and the spaces

$$
X_{k s}^{1}=\left\{f(t) \in X_{k s} \mid \dot{f}(t) \in X_{k s}\right\},\|f(t)\|_{X_{k s}^{1}}=\max \left\{\|f(t)\|_{X_{k s}},\|\dot{f}(t)\|_{X_{k s}}\right\} .
$$

Everywhere below the operator $\stackrel{\sqcap}{A}^{-1} \stackrel{\sqcap}{B}$ is supposed to be bounded in $X_{k 1}$ (for the case $\mathrm{k}=1$ it is evident). Then the operator

$$
\mathbf{A} x=A \dot{x}-B x
$$

acting from $X_{k 0}^{1}$ to $Y_{k 0}$ is linear and continuous with $X_{k 2} \subset \mathcal{N}(\mathbf{A})$.

Let be $D_{k} \supset S_{k}=$ \{initial values of solutions of the equation (8), which are defined and remain in a small neighborhood of zero in $D_{k}$ for $\left.t \in[0,+\infty)\right\}$ 
and $U_{k}=$ initial values of solutions of (8), which are defined and remain in a small neighborhood of zero in $D_{k}$ for $\left.t \in(-\infty, 0]\right\}$. From (11) it follows that

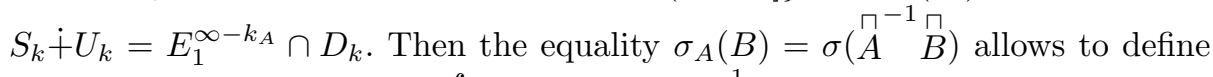

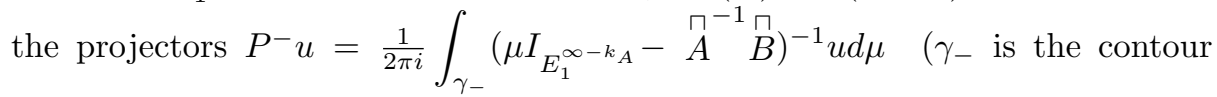
in $\rho_{A}(B)$ containing inside itself the points $\mu \in \sigma_{A}(B)$ with $\operatorname{Re} \mu<0$ ), and $P^{+}=I_{E_{1}^{\infty-k_{A}}}-P^{-}$. Whence $D_{k}=D_{k}^{-} \dot{+} D_{k}^{0} \dot{+} D_{k}^{+}, D_{k}^{0}=E_{1}^{k_{A}}, D_{k}^{ \pm}=P^{ \pm} D_{k}$. Operator $\mathbf{A}$ is Noetherian [5] with $R(\mathbf{A})=Y_{k 1}$ and

$$
\begin{aligned}
& \mathcal{N}(\mathbf{A})=\left\{f(t) \in X_{k 0}^{1} \mid f(t)=\exp \left(\stackrel{\sqcap}{A}^{-1} \stackrel{\sqcap}{B} t\right) P^{-} f(0) \in D_{k}^{-}\right\} \dot{+}\left\{f(t) \in D_{k}^{0}\right\} \\
& =\mathcal{N}_{1}(\mathbf{A}) \dot{+} \mathcal{N}_{2}(\mathbf{A}) \text { for } t \geq 0 \\
& \left(\mathcal{N}(\mathbf{A})=\left\{f(t) \in X_{k 0}^{1} \mid f(t)=\exp \left(\stackrel{\sqcap}{A}^{-1} \stackrel{\sqcap}{B} t\right) P^{+} f(0) \in D_{k}^{+}\right\} \dot{+}\left\{f(t) \in D_{k}^{0}\right\}\right. \\
& \text { for } t \leq 0) \text {. }
\end{aligned}
$$

Now setting $x=y+z+v, z \in D_{k}^{+}, v \in D_{k}^{0}=E_{1}^{k_{A}}, y \in D_{k}^{-}$one can write the equation (1) in the form $(w=y+z$ in (9) $)$

$$
\mathbf{A} z=R(z+y+v) \quad(\mathbf{A} y=R(y+z+v))
$$

and apply the implicit operator theorem to (12) regarding $y, v(z, v)$ as functional parameters (see the relevant theorems 22.1 and 22.2 in [5] for continuous and analytic operator $R$ respectively). It follows that (12) has a sufficiently smooth or analytic (according to the properties of the operator $R$ ) solution in some neighborhoods of parameters $y, v(z, v)$ zero values

$$
z=z(y+v), \quad z(0)=0=D z(0) \quad(y=y(z+v), y(0)=0=D y(0))
$$

Consequently it is true the following Grobman-Hartman theorem [1] analogue asserting that the local solutions behavior for nonlinear equation in hyperbolic equilibrium neighborhood is the same that for its linearization.

Theorem 1. There exist a neighborhood $\omega^{-}\left(\omega^{+}\right)$of zero in $D_{k}^{0} \dot{+} D_{k}^{-}$(in $\left.D_{k}^{0} \dot{+} D_{k}^{+}\right)$and sufficiently smooth mapping $z_{R}=z_{R}(\xi, \eta)=z_{R}(\xi \cdot \phi+\eta)$ : $\omega^{-} \rightarrow D_{k}^{+}, \eta \in D_{k}^{-}\left(y_{R}=y_{R}(\xi, \zeta)=y_{R}(\xi \cdot \phi+\zeta): \omega^{+} \rightarrow D_{k}^{-}, \zeta \in D_{k}^{+}\right)$, such that a) $z_{R}(0,0)=0, D_{\xi} z_{R}(0,0)=0, D_{\eta} z_{R}(0,0)=0\left(y_{R}(0,0)=0\right.$, $\left.D_{\xi} y_{R}(0,0)=0, D_{\zeta} y_{R}(0,0)=0\right)$, b) for any solution $x(t)$ of (1) with initial data $x(0)=\xi \cdot \phi+\eta+z_{R}(\xi \cdot \phi+\eta)\left(x(0)=\xi \cdot \phi+y_{R}(\xi \cdot \phi+\zeta)+\zeta\right)$ one has $z(t)=z_{R}(\xi(t) \cdot \phi+y(t)) \in D_{k}^{+}$for $t \geq 0\left(y(t)=y_{R}(\xi(t) \cdot \phi+z(t)) \in D_{k}^{-}\right.$ for $t \leq 0)$, c) any solution $x(t)$ of (1) with initial data from b) takes the form $x(t)=\xi(t) \cdot \phi+y(t)+z_{R}(\xi(t) \cdot \phi+y(t))\left(x(t)=\xi(t) \cdot \phi+y_{R}(\xi(t) \cdot \phi+z(t))+z(t)\right)$ and tends to zero when $t \rightarrow+\infty(t \rightarrow-\infty)$, and belongs, consequently, to local stable manifold $S_{k}(R)$ (local unstable manifold $U_{k}(R)$ ).

Proof. We give here the proof for the function $z_{R}$ and local stable manifold $S_{k}(R)$, the proof of the second part is analogous. Define the projector $\tilde{P}^{-}$of 
$X_{k 1}^{1}$ onto $\mathcal{N}_{1}(\mathbf{A})$ by the equality $\left(\tilde{P}^{-} f\right)(t)=\exp \left(\stackrel{\sqcap^{-1}}{\stackrel{\sqcap}{B}} t\right) P^{-} f(0), t \geq 0$. If one sets $x(t)=v(t)+y(t)+z(t), v(t)=\mathbf{p} x(t), v(0)=\xi \cdot \phi=\sum_{i=1}^{m} \sum_{s=1}^{q_{i}} \xi_{i s}$. $\phi_{i}^{(s)}, y(t)=\tilde{P}^{-} x(t)=\exp \left(\stackrel{\sqcap}{A}^{-1} \stackrel{\sqcap}{B} t\right) \eta, \eta=y(0), z(t)=\left(I_{X_{k 1}^{1}}-\tilde{P}^{-}\right) x(t)$, then the Lyapounov-Schmidt method (Theorem 27.1 [5] for Noetherian operators with $d$-characteristic $(n, 0)$ and the indicated above theorems 22.1, $22.2[5])$ implies there is a unique solution of (12) $z=z_{R}(\xi(t) \cdot \phi+y(t)) \in X_{k 1}^{1}$, such that $x(0)=\xi \cdot \phi+\eta+z_{R}(\xi \cdot \phi+\eta)$, i.e. the unique solution of $(1) x(t)=v(t)+y(t)+$ $z_{R}(\xi(t) \cdot \phi+y(t)), v(t)=\xi(t) \cdot \phi$, in a sufficiently small semi-neighborhood of $t=0$, where the function $z_{R}(\xi, \eta)=z_{R}(\xi \cdot \phi+\eta)$ is sufficiently smooth by $\xi, \eta$, and $z_{R}(0,0)=0, D_{\xi} z_{R}(0,0)=0, D_{\eta} z_{R}(0,0)=0$.

Writing the equation (1) in $\mathbf{p}, \mathbf{q}$-projections at the usage of the theorem 1 one can get the system for the determination of $\xi_{i s}(t)$ (so-named the resolving system (RS) for the equation (1) $1210[14])$. Here $x(t)=\xi(t) \cdot \phi+w(t)$, where $w(t)=y(t)+z_{R}(\xi(t) \cdot \phi+y(t))$ for $t \geq 0$ and $w(t)=y_{R}(\xi(t) \cdot \phi+z(t))+z(t)$ for $t \leq 0$

$$
\begin{gathered}
\sqcap \frac{d w}{d t}=\stackrel{\sqcap}{B} w-\left(I_{D_{k}}-\mathbf{q}\right) R(\xi \cdot \phi+w) \\
0=\xi_{i q_{i}}(t)-<R(\xi(t) \cdot \phi+w), \hat{\psi}_{i}^{(1)}>, \\
\dot{\xi}_{i q_{i}}(t)=\xi_{i, q_{i}-1}(t)-<R(\xi(t) \cdot \phi+w), \hat{\psi}_{i}^{(2)}>, \\
\ldots \ldots \ldots \ldots \ldots \ldots \ldots \ldots \ldots \ldots \ldots \ldots \ldots \ldots \\
\dot{\xi}_{i 2}(t)=\xi_{i 1}(t)-<R(\xi(t) \cdot \phi+w), \hat{\psi}_{i}^{\left(q_{i}\right)}>, \\
\xi_{i s}(0)=\xi_{i s}, \quad s=1, \ldots, q_{i}, \quad i=1, \ldots, m .
\end{gathered}
$$

Consequently, the manifold $S_{k}(R)=\{$ initial values of solutions of the equation (1), which are defined and remain in a small neighborhood of $0 \in D_{k}$ for $t \in$ $[0,+\infty)\}$ (the manifold $U_{k}(R)=\{$ initial values of solutions (1), which are defined and remain in a small neighborhood of $0 \in D_{k}$ for $\left.\left.t \in(-\infty, 0]\right\}\right)$ has the form $x(0)=\xi \cdot \phi+\eta+z_{R}(\xi \cdot \phi+\eta)\left(x(0)=\xi \cdot \phi+y_{R}(\xi \cdot \phi+\zeta)+\zeta\right)$, where $\eta \in D_{k}^{-}\left(\zeta \in D_{k}^{+}\right)$and $\xi$ are small.

Remark 1. Determined by the function $\xi \cdot \phi+\eta+z_{R}(\xi \cdot \phi+\eta)$ for $t \geq 0\left(\xi \cdot \phi+y_{R}(\xi\right.$. $\phi+\zeta)+\zeta$ for $t \leq 0)$ invariant manifold $\mathfrak{M}$ can be regarded as center manifold $\left(\xi \cdot \phi \in D_{k}^{0}\right)$, that is nontrivial for the equation (1) even if $\left\{\mu \in \sigma_{A}(B) \mid \operatorname{Re} \mu=\right.$ $0\}=\varnothing$. Here $\{\xi \cdot \phi\}$ can be named as linear center manifold tangent to $\mathfrak{M}$. One can say that $\mathfrak{M}$ has an hyperbolic structure. Thus the RS (15) represents the differential-algebraic system on $\mathfrak{M}$. Of course, if the operator $A$ is invertible, $\mathfrak{M}$ and the system (15) are absent, i.e. in the Grobman-Hartman theorem $z_{R}=$ $z_{R}(\eta)$ [1].

Theorem 2. Let the operators $A, B$ and $R$ in (1) be intertwined by the group $G$ representations $L_{g}$ (acting in $E_{1}$ ) and $K_{g}$ (acting in $E_{2}$ ) and the condition $I$ (direct supplements $E_{1}^{\infty-m}$ to $\mathcal{N}(A)$ and $E_{1}^{\infty-n}$ to $\mathcal{N}(B)$ are invariant relative to $\left.L_{g}\right)$ is satisfied. Then the center manifold $\mathfrak{M}$ is invariant relative to the operators $L_{g}$. 
Proof. According to [14 projectors $\mathbf{p}, \mathbf{P}(\mathbf{q}, \mathbf{Q})$ commute with the operators $L_{g}\left(K_{g}\right)$ and invariant pairs of subspaces reduce the representations $L_{g}\left(K_{g}\right)$.

In the article [10] it is proved that the stability (instability) of the trivial solution (even for non-autonomous) equation (1) at sufficiently general conditions is determined by the RS (15) with corollaries for the investigation of the stability (instability) of bifurcating solutions.

It is interesting the case when $\sigma_{A}^{+}(B)=\varnothing$. Then $D_{k}=D_{k}^{-} \dot{+} D_{k}^{0}, x(t)=$ $\xi(t) \cdot \phi+y(t)$ and the center manifold has the form $\xi(t) \cdot \phi+y(\xi(t) \cdot \phi)$. Here the equation (14) gives

$$
\begin{gathered}
\sqcap y^{\prime}(\xi(t) \cdot \phi)\left(\frac{d \xi}{d t} \cdot \phi\right)=\stackrel{\sqcap}{B} y(\xi(t) \cdot \phi)+(I-\mathbf{q}) R(\xi(t) \cdot \phi+y(\xi(t) \cdot \phi)), \\
y(0)=0, \quad y^{\prime}(0)=0
\end{gathered}
$$

In combination with (15) this gives a possibility for the determination of center manifold $w(\xi(t) \cdot \phi)=\xi(t) \cdot \phi+y(\xi(t) \cdot \phi)$ by successive approximations in conditions of sufficiently smooth operator $y(\xi \cdot \phi)$. However on this way essential difficulties arise connected with the fact that the system (15) is differentialalgebraic, i.e. the differential equations for the functions $\xi_{i 1}(t), i=1, \ldots, m$, are absent. One can find $y(\xi \cdot \phi)$ iteratively at the differentiation of the first equations (15).

Remark 2. Theorem 1 and all corollaries remain true for the parameter depending equation

$$
A \frac{d x}{d t}=B x-R(x, \lambda), \quad R(0, \lambda) \equiv 0, \quad R_{x}(0,0)=0,
$$

( $\lambda \in \Lambda, \Lambda$ is some Banach space) in a small neighborhood of $\lambda=0$, when as earlier $\operatorname{Re} \sigma_{A}(B) \neq 0$, i.e. $\lambda=0$ is not a bifurcation point. However all functions $w, z_{R}$ and $y_{R}$ will depend on small parameter $\varepsilon$.

\section{One Case of $\sigma_{A}^{0}(B) \neq \varnothing$}

Here it is considered the simplest case when $\sigma_{A}^{+}(B)=\varnothing$, but $\sigma_{A}^{0}(B)=\{\mu \in$ $\left.\sigma_{A}(B) \mid R e \mu=0\right\} \neq \varnothing$ contains some finite number $2 n=2 n_{1}+\ldots+2 n_{\ell} \mathrm{A}$ eigenvalues $\pm i \alpha_{s}$ of multiplicities $n_{s}, s=1, \ldots, \ell, \alpha_{s}=\kappa_{s} \alpha, \alpha \neq 0$ with coprime $\kappa_{s}>0$ or (and) zero-eigenvalue. Without loss of generality it is supposed that the equation (1) is written in the form of the system

$$
\begin{aligned}
& A_{1} \dot{x}=B_{1} x-f(x, y) \\
& A_{2} \dot{y}=B_{2} y-R(x, y),
\end{aligned}, A=\left(\begin{array}{cc}
A_{1} & 0 \\
0 & A_{2}
\end{array}\right), B=\left(\begin{array}{cc}
B_{1} & 0 \\
0 & B_{2}
\end{array}\right)
$$

where the linear operators $A_{1}, B_{1}: E_{1}^{k_{B_{1}}} \rightarrow E_{2, k_{B_{1}}}\left(k_{B_{1}}=2 n_{1} p_{1}+\ldots+2 n_{\ell} p_{\ell}\right.$, $p_{s}$ are $A_{1}$-Jordan chains lengths for $\left.\pm i \alpha_{s}, s=1, \ldots, \ell\right)$ act in the invariant pair of finite dimensional subspaces $E_{1}^{k_{B_{1}}}, E_{2, k_{B_{1}}}$ and $A_{2}, B_{2}$ act in the invariant pair of subspaces $E_{1}^{\infty-k_{B_{1}}}, E_{2, \infty-k_{B_{1}}}$. Thus, $\sigma_{A_{1}}\left(B_{1}\right)=\sigma_{A}^{0}(B)$ and $\sigma_{A_{2}}^{0}\left(B_{2}\right)=\varnothing$. 
Here $f$ and $R$ are $C^{2}$-functions vanishing together with their first derivatives at the origin.

The main assumption in the simplest case is

$$
\mathcal{N}\left(A_{1}\right)=\{0\}, \quad \mathcal{N}\left(A_{2}\right)=\operatorname{span}\left\{\phi_{(2) 1}, \ldots, \phi_{(2) m_{2}}\right\}
$$

Then under section 3 conditions there exists the function $y_{R}\left(\xi_{2}(t) \cdot \phi_{(2)}, x\right)$ vanishing together with its first derivatives at the origin, such that the second equation (18) is reducing to the system

$$
\stackrel{\sqcap}{A}_{2} \frac{d y_{R}}{d t}=\stackrel{\sqcap}{B}{ }_{2} y_{R}-\left(I-\mathbf{q}_{(2)}\right) R\left(x, \xi_{2}(t) \cdot \phi_{(2)}+y_{R}\left(\xi_{2}(t) \cdot \phi_{(2)}, x\right)\right)
$$

$\left(\mathbf{q}_{(2)}=\sum_{i=1}^{m_{2}} \sum_{j=1}^{q_{2, i}}<\cdot, \hat{\psi}_{(2), i}^{(j)}>\zeta_{(2)}^{(j)}: E_{2, \infty-k_{A_{2}}} \rightarrow \operatorname{span}\left\{\zeta_{(2) i}^{(j)}\right\}, \stackrel{\sqcap}{A}_{2}, \stackrel{\sqcap}{B}_{2}\right.$ act in invariant pair of subspaces $E_{1}^{\infty-k_{B_{1}}-k_{A_{2}}}, E_{2, \infty-k_{B_{1}}-k_{A_{2}}}$ )

$$
\begin{aligned}
& 0=\xi_{2 i q_{2, i}}(t)-<R\left(x, \xi_{2}(t) \cdot \phi_{(2)}+y_{R}\left(\xi_{2}(t) \cdot \phi_{(2)}, x\right)\right), \hat{\psi}_{(2), i}^{(1)}>, \\
& \dot{\xi}_{2 i q_{2, i}}(t)=\xi_{2 i, q_{2, i}-1}(t)-<R\left(x, \xi_{2}(t) \cdot \phi_{(2)}+y_{R}\left(\xi_{2}(t) \cdot \phi_{(2)}, x\right)\right), \hat{\psi}_{(2), i}^{(2)}> \\
& \dot{\xi}_{2 i 2}(t)=\xi_{2 i 1}(t)-<R\left(x, \xi_{2}(t) \cdot \phi_{(2)}+y_{R}\left(\xi_{2}(t) \cdot \phi_{(2)}, x\right)\right), \hat{\psi}_{(2), i}^{\left(q_{2, i}\right)}>, \\
& \xi_{2 i \sigma}(0)=\xi_{2 i \sigma}, \sigma=1, \ldots, q_{2, i}, \quad i=1, \ldots, m_{2} \text {. }
\end{aligned}
$$

If the system (18) is equipped with initial values $x(0), y(0)$, then they must satisfy the equality

$$
y(0)=\xi_{2} \cdot \phi_{(2)}+y_{R}\left(\xi_{2} \cdot \phi_{(2)}, x(0)\right) .
$$

Now one has to solve the problem

$$
A_{1} \dot{x}=B_{1} x-f\left(x, \xi_{2}(t) \cdot \phi_{(2)}+y_{R}\left(\xi_{2}(t) \cdot \phi_{(2)}, x\right)\right)
$$

at the initial data $x(0)$ satisfying (22).

Thus one has two systems (21) and (23) on the center manifold $y=y_{R}\left(\xi_{2}(t) \cdot \phi_{(2)}, x\right)$.

\section{Grobman-Hartman Theorem Analog for Maps}

According to section 3 the equation (14) can be written in the form

$$
\frac{d w}{d t}=\stackrel{\sqcap^{-1}}{A} \stackrel{\sqcap}{B} w-\stackrel{\sqcap}{A}^{-1}\left(I_{D_{k}}-\mathbf{q}\right) R(\xi \cdot \phi+w)
$$

in the space $X_{k 1}^{1}$. Then the assumption about the boundedness of the operator $\sqcap^{-1} \sqcap$

$A \quad B$ in $X_{k 1}$ allows to prove Grobman-Hartman theorem for maps [15]. In fact, then for small $\xi$ there exists the resolving operator $U_{\xi}(t, \cdot): X_{k 1} \rightarrow X_{k 1}^{1}, w_{0} \mapsto$ $w(t)$ for the problem (24) with the initial value $w(0)=w_{0}$ (at $\xi=0, U_{0}(t)$ is linear). Thus the following assertion is true: 
Theorem 3. For small $\xi$ at $\sigma_{A}^{0}(B)=\varnothing$ and operator $\stackrel{\sqcap^{-1}}{A} \stackrel{\sqcap}{B}$ boundedness assumption there exits the resolving operator $U_{\xi}\left(t, w_{0}\right)$ and a homeomorphism $\Phi_{\xi}: X_{k 1}^{1} \rightarrow X_{k 1}^{1},\|\xi\| \ll 1$, such that for $t \in R$ and $w_{0} \in X_{k 1}$ the following relation

$$
U_{0}(t) \Phi_{\xi}\left(w_{0}\right)=\Phi_{\xi}\left(U_{\xi}\left(t, w_{0}\right)\right)=\Phi_{\xi}(w(t))
$$

is true, where the function $w(t)$ and the initial values $w_{0}, \xi_{0}$ satisfy the initial value problem for differential-algebraic system (15).

Remark 3. The results of this article remains true for the more general operators subordinateness ( $A$ is subordinate to $B$ if on $D_{B}\|A x\| \leq\|B x\|+\alpha\|x\|, \alpha \geq 0$ ).

The authors are thankful to Prof. V.S. Mokeychev (Kazan' State University) for this remark on improvement of our article.

\section{References}

1. Gurel, O. (Ed.): Bifurcation Theory and its Applications in Scientific Disciplines. Annals of the New York Academy of Sci. Vol. 316. (1979)

2. Joseph, D.J.: Stability of Fluid Motions. Springer Verlag (1976)

3. Henry, D.: Geometric Theory of Semilinear Parabolic Equations. Lect. Notes in Math, Vol. 840. Springer Verlag (1981)

4. Govaerts, W.J.F.: Numerical Methods for Bifurcations of Dynamical Equilibria. SIAM, Philadelfia (2000)

5. Vainberg, M., Trenogin, V.: Branching Theory of Solutions of Nonlinear Equations. Wolters Noordorf, Leyden (1974)

6. Carr, J.: Applications of Centre Manifold Theory. Appl. Math. Sci. Vol. 35. Springer Verlag (1981)

7. Barenblatt, G.I., Zheltov, Yu.P., Kochina, I.N.: On the principal conceptions of the filtration theory in jointing media. Appl. Math. Mech. Vol. 24.5 (1960) 58-73

8. Whitham, G.B.: Linear and Nonlinear Waves. Wiley-Int. Publ. (1974)

9. Oskolkov, A.P.: Initial-boundary value problems for equations of Kelvin-Foight and Oldroidt fluids. Proc. Steklov Math. Inst. AN SSSR. Vol. 179 (1988) 126-164

10. Loginov B., Rousak, Ju.: Generalized Jordan structure in the problem the stability of bifurcating solutions. Nonlinear Anal. TMA. Vol. 17. 3 (1991) 219-232

11. Hale, J.: Introduction to dynamic bifurcation. Bifurcation Theory and Appl. Lect. Notes in Math. Vol. 1057. Springer Verlag (1984) 106-151

12. Loginov, B.: Branching equation in the root subspace. Nonlinear Anal. TMA. Vol. 32.3 (1998) 439-448

13. Sviridiuk, G.: Phase spaces of semilinear Sobolev-type equations with relatively strong sectorial operator. Algebra and Anal. Vol. 6. 5 (1994) 252-272

14. Loginov, B., Konopleva, I.: Symmetry of resolving systems for differential equations with Fredholm operator at the derivative. Proc. of Int. Conf. MOGRAN-2000 Ufa. USATU (2000) 116-119

15. Volevich, L., Shirikyan, A.: Local dynamics for high-order semilinear hyperbolic equations. Izvestya RAN. Vol. 64.3 (2000) 439-485 